\title{
A shift sequence based approach for nurse scheduling and a new benchmark dataset
}

\author{
Peter Brucker • Edmund K. Burke • Tim Curtois • \\ Rong Qu • Greet Vanden Berghe
}

Received: 21 March 2006 / Revised: 6 November 2007 / Accepted: 9 October 2008

(C) Springer Science+Business Media, LLC 2008

\begin{abstract}
This paper investigates an adaptive constructive method for solving nurse rostering problems. The constraints considered in the problems are categorised into three classes: those that are sequence related, those that are nurse schedule related and those that are roster related. We propose a decomposition approach (to construct solutions) that consists of two stages: (1) to construct high quality sequences for nurses by only considering the sequence constraints, and (2) to iteratively construct schedules for nurses and the overall rosters, based on the sequences built and considering the schedule and roster constraints. In the second stage of the schedule construction, nurses are ordered and selected adaptively according to the quality of the schedules they were assigned to in the last iteration. Greedy local search is carried out during and after the roster construction, in order to improve the (partial) rosters built. We show that the local search heuristic during the roster construction can further improve the constructed solutions for the benchmark problems tested.

In addition, we introduce new benchmark nurse rostering datasets which are based upon real world data. The data sets represent a variety of real world constraints. The publication of this problem data to the research community is aimed at closing the gap
\end{abstract}

P. Brucker

Fachbereich Mathematik/Informatik, University of Osnabruck, Albrechtstr. 28, 49069 Osnabruck, Germany

E.K. Burke · T. Curtois · R. Qu (凶)

Automated Scheduling, Optimisation and Planning (ASAP) Group, School of Computer Science, University of Nottingham, Nottingham, NG8 1BB, UK

e-mail: rxq@cs.nott.ac.uk

G. Vanden Berghe

Information Technology, Engineering Department, KaHo St.-Lieven, 9000 Gent, Belgium

G. Vanden Berghe

Department of Computer Science, K.U. Leuven campus Kortrijk, E. Sabbelaan 53, 8500 Kortrijk, Belgium 
between theory and practice in nurse scheduling research. One of the main objectives is to encourage more research on these data sets.

Keywords Adaptive heuristic $\cdot$ Benchmark $\cdot$ Nurse rostering $\cdot$ Shift sequence

\section{Introduction}

Nurse rostering problems consist of allocating the required workload to nurses subject to a number of constraints (see Burke et al. 2004c; Ernst et al. 2004; Sitompul and Randhawa 1990). The common objective of nurse rostering is to efficiently utilise resources and thus to produce rosters with a balanced workload as well as to satisfy individual preferences as much as possible.

Constraints are usually categorised into two groups: hard and soft constraints, which vary significantly with respect to legal regulations and individual preferences, depending on individual institutions and countries. Hard constraints must be satisfied to obtain feasible solutions. Soft constraints are desirable but not obligatory, and thus can be violated. In real nurse rostering settings, we noticed that the problems are nearly always over-constrained. It is therefore quite common to express the quality of solutions in terms of soft constraint violations.

Early research in nurse rostering was overviewed in Pierskalla and Rath (1976), and Warner et al. (1990). These early approaches were effective in solving small scale problems but were not flexible and were too computationally expensive to deal with large problems with many constraints.

Meta-heuristics have been widely studied in nurse rostering over the past two decades or so and have had significant success for a range of problems (see Burke et al. 2004c). Relevant approaches include Tabu Search (Burke et al. 1999; Dowsland 1998; Valouxis and Housos 2000), Simulated Annealing (Brusco and Jacobs 1995; Jaszkiewicz 1997), Evolutionary Algorithms (Aickelin and Dowsland 2000, 2003; Burke et al. 2001; Jan et al. 2000; Post et al. 2004) and Variable Neighbourhood Search (Brucker et al. 2005; Burke et al. 2004a, 2008). Hybrid techniques, particularly between meta-heuristics and mathematical programming methods have formed an important part of the nurse rostering literature (see Bard and Purnomo 2005, 2007; Beaumont 1997). Artificial intelligence approaches such as case-based reasoning (Beddoe and Petrovic 2006; Petrovic et al. 2003; Scott and Simpson 1998), constraint-based techniques (Adbennadher and Schlenker 1999; Jaumard et al. 1998; Meisels et al. 1996; Meyer auf'm Hofe 2000) and expert systems (Chen and Yeung 1993) have also been investigated with some success.

Most nurse rostering models apply hard and soft constraints. However, in Ikegami and Niwa (2003), constraints were categorised into shift constraints (which concerned the number of staff and the skill category required for each shift), and nurse constraints (which considered the workload for each nurse including nurse preferences, consecutive shifts and intervals between shifts). The nurse constraints were used to produce all feasible shift patterns of the whole scheduling period for each nurse, independently from shift constraints. The best combinations of these shift patterns were found using mathematical programming and metaheuristics (Ikegami and Niwa 2003). 
In Scott and Simpson (1998), a decision support system framework was presented, in which a pattern database, a schedule database and a nurse database were employed to address nurse rostering problems. Shift patterns were produced for one week and a screening process was used to eliminate undesirable ones. Low penalty patterns were combined to generate cyclic work schedules using a local search method. Only cyclic schedules of complete weeks were addressed in the decision support system.

In Aickelin and Dowsland (2003), all the feasible weekly shift patterns were predefined and associated with costs concerning preferences, requests, the number of successive days, etc. These (in total 411) shift patterns were then used to construct nurse rosters by employing different heuristic decoders within a genetic algorithm to schedule both shifts and patterns for the best permutations of nurses. The idea of permuting the nurses to be scheduled is similar to the method presented in this paper. Two types of shifts (Night and Day) were addressed and the pre-construction of patterns required a large amount of time. In Aickelin and Li (2007), exactly the same problems were solved by using a Bayesian network, which explicitly learned the rules for selecting the patterns to be assigned to the nurses. The results, compared with those obtained in Aickelin and Dowsland (2003), indicated the strength of the approach.

Valouxis and Housos (2000) explored workstretch in an Integer Linear Programming model. Extra constraints on the minimum and maximum lengths of the workstretch were introduced to simplify the model so that only feasible workstretch was tackled.

Mason and Smith (1998) introduced workstretch cost and workstretch transition in an Integer Programming model to define the cost of the day-on within and between the workstretch. Column generation was employed to decide on the content of the workstretch and to link them in constructing the schedules concerning other costs related to shifts.

The approach in this paper draws upon that of Ikegami and Niwa (2003), where a concept called stint is introduced to define a feasible sequence of shifts on consecutive days. Schedules for nurses can then be constructed by using a series of stints. Millar and Kiragu (1998) used the term stint to denote patterns, which were defined by a start date, a length, a cost and the shifts. Network programming was used where each node is a stint to construct either cyclic or non-cyclic rosters.

In our previous work (Brucker et al. 2005), high quality pre-defined schedules have been employed to construct cyclic schedules for a group of nurses with the same requirements. Based on these partial cyclic schedules, the rest of the shifts were assigned to the rest of the nurses with different requirements. The problems can thus be seen as being decomposed into cyclic and non-cyclic parts. A Variable Neighbourhood Search developed in Burke et al. (2008) was used to further improve the generated rosters. Preliminary results indicated promising directions on high quality pre-defined schedules.

In this work, we further explore this idea by considering patterns, which are sequences of shifts in constructing nurse schedules and rosters. The generated rosters contain non-cyclic schedules, which are applicable to more general real-world problems (Burke et al. 2004c). We consider patterns rather than individual shifts (as in many other nurse rostering models) for constructing the rosters. While nurses' 
schedules can be seen as shift sequences within the scheduling period, the issues of shift patterns and workstretch have received far less attention in nurse rostering (see above).

In Sect. 2, we present the problem formulation. The two-stage approach and the benchmark problems are described in Sects. 3 and 4, respectively. Section 5 reports experimental results and addresses important issues concerning adaptation. Finally, we present conclusions and future work in Sect. 6.

\section{Problem formulation}

A solution of the nurse rostering problem consists of a collection of personal schedules for each of the nurses. A schedule for a nurse usually consists of shift sequences that possibly have different lengths and different types (i.e. night shifts, early shifts). The shifts in a sequence must be performed on consecutive days, one shift per day. Between the sequences in a schedule (which are called day-on periods) there are day-off periods, i.e. days without shifts.

Accordingly, we define the following terms that are frequently used in the rest of the paper:

- By sequence we mean a series of shifts for nurses. A sequence penalty indicates the quality of the sequence. A set of sequences is associated with each nurse.

- A schedule is an ordered list of sequences and day-off periods for a single nurse of a certain skill category. A schedule penalty indicates each schedule's quality. The length of a schedule is equal to the total number of days-off and days-on in the scheduling period.

- A roster describes the overall solution. It consists of schedules of the same length, one for each nurse. The quality of the roster is determined by the violations of the constraints.

We categorise the constraints as sequence, schedule and roster constraints, which are listed below:

- Sequence constraints are applied within shift sequences (i.e. to construct shift sequences and to evaluate their quality) for each nurse with certain skills (i.e. each nurse is associated with only those valid sequences satisfying constraint No. 1 in Table 1).

- Schedule constraints are applied to combine shift sequences (i.e. to construct schedules and evaluate their quality) for each nurse.

Table 1 Hard constraints (Burke et al. 2004b) categorised into sequence and roster constraints

\begin{tabular}{llr}
\hline & Hard constraint & Type \\
\hline 1 & $\begin{array}{l}\text { Shifts which require certain skills can only be taken by (or assigned to) } \\
\text { nurses who have those skills }\end{array}$ & sequence \\
2 & The shift coverage requirements must be fulfilled & roster \\
\hline
\end{tabular}


- Roster constraints are applied when combining nurse schedules (i.e. to construct an overall solution) while satisfying the coverage requirements on each day.

Furthermore, hard constraints and soft constraints are presented as follows:

- The hard constraints considered in this research are listed in Table 1. The last column in this and the next table indicates the constraint type used in our approach (i.e. sequence, schedule or roster constraints).

- The soft constraints are listed in Table 2. They represent different problem requirements and nurse preferences. Note that this is a complete constraint list representing all the possible constraints considered in the benchmark problems. Different problem instances presented in Sect. 4 may have different subsets of these constraints.

Table 2 Soft constraints (Burke et al. 2004b) categorised into sequence, schedule and roster constraints

\begin{tabular}{|c|c|c|}
\hline & Soft constraint & Type \\
\hline 1 & Minimum rest time between shifts & sequence \\
\hline 2 & $\begin{array}{l}\text { Alternative skill (if a nurse is able to cover a shift but prefers not to as it does } \\
\text { not require his/her primary skill) }\end{array}$ & sequence \\
\hline 3 & Maximum number of shift assignments & schedule \\
\hline 4 & Maximum number of consecutive working days & sequence \\
\hline 5 & Minimum number of consecutive working days & sequence \\
\hline 6 & Maximum number of consecutive non-working days & schedule \\
\hline 7 & Minimum number of consecutive non-working days & schedule \\
\hline 8 & Maximum number of hours worked & schedule \\
\hline 9 & Minimum number of hours worked & schedule \\
\hline 10 & $\begin{array}{l}\text { Maximum total number of assignments for all Mondays, Tuesdays, } \\
\text { Wednesdays, etc. }\end{array}$ & schedule \\
\hline 11 & $\begin{array}{l}\text { Maximum number of a certain shift type worked (e.g. maximum seven night } \\
\text { shifts for the planning period) }\end{array}$ & schedule \\
\hline 12 & $\begin{array}{l}\text { Maximum number of a certain shift type worked per week (same as above but } \\
\text { for each individual week) }\end{array}$ & schedule \\
\hline 13 & Valid number of consecutive shifts of the same type & sequence \\
\hline 14 & Free days after night shifts & schedule \\
\hline 15 & $\begin{array}{l}\text { Complete weekends (i.e. shifts on both Saturday and Sunday, or no shift over } \\
\text { the weekend) }\end{array}$ & schedule \\
\hline 16 & No night shifts before free weekends & schedule \\
\hline 17 & Identical shift types during the weekend & schedule \\
\hline 18 & Maximum number of consecutive working weekends & schedule \\
\hline 19 & Maximum number of working weekends in four weeks & schedule \\
\hline 20 & Maximum number of working bank holidays & schedule \\
\hline 21 & Shift type successions (e.g. Is shift type A allowed to follow B the next day, etc.) & sequence \\
\hline 22 & Requested days on or off & schedule \\
\hline 23 & Requested shifts on or off & schedule \\
\hline 24 & Tutorship (employee $\mathrm{X}$ present when employee $\mathrm{Y}$ is working) & roster \\
\hline 25 & Working separately (employee $\mathrm{X}$ not present when employee $\mathrm{Y}$ is working) & roster \\
\hline
\end{tabular}




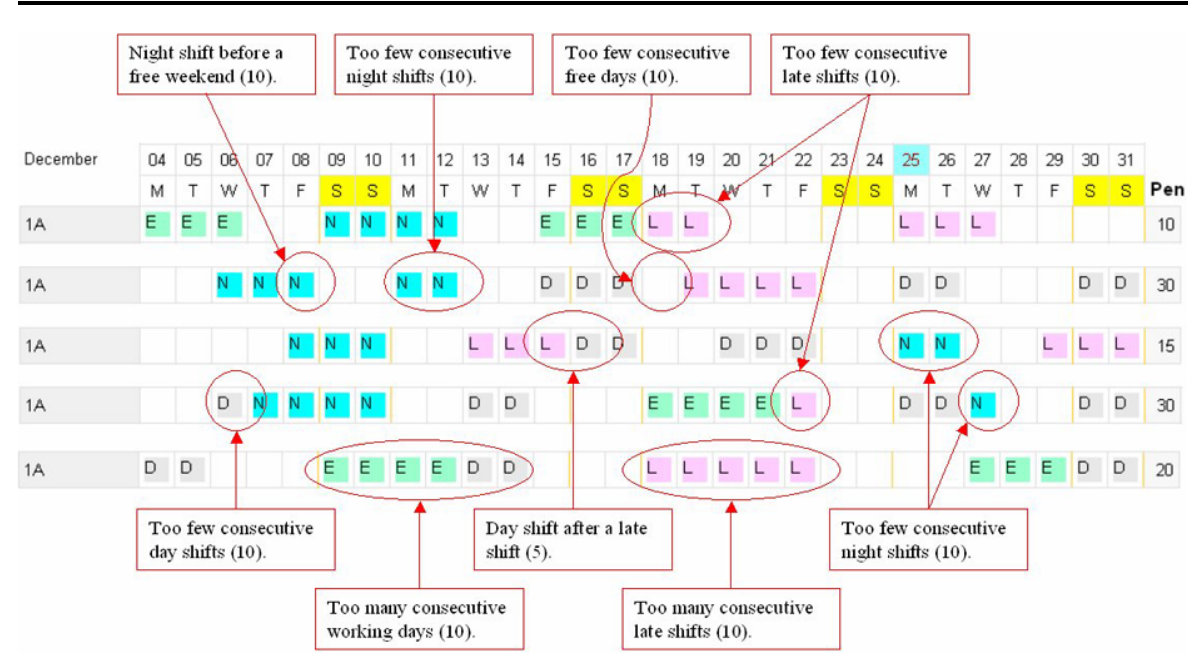

Fig. 1 Example schedules for employee 1A

Hard constraint No. 1 in Table 1 is considered when constructing shift sequences for each nurse of different skills. For example, 'DH', which denotes the day shift for a head nurse, is only applied when constructing shift sequences for a head nurse with a certain skill level. Hard constraint No. 2 states that the total number of shifts on certain days must satisfy the coverage requirements (i.e. we require enough shifts of a certain type every day). Violations of soft constraints in the solutions are penalised by certain values and are used for evaluating the quality of the rosters produced. The objective is to find feasible solutions that satisfy the soft constraints as much as possible (i.e. minimising the overall penalty of nurses' schedules in the roster).

We present, in Fig. 1, a few possible schedules with their associated penalties for an example employee for a four-week period in December. Table 3 contains some of the constraints with their penalties for this employee. Table 4 provides details of the shifts referred to in these constraints in Table 3. For example, the valid length of consecutive late shift $\mathrm{L}$ and night shift $\mathrm{N}$ (see Table 4 for the definition of L and $\mathrm{N}$ ) is either 3 or 4 , respectively (constraint No. 8 in Table 3 ).

\section{A two-stage adaptive approach}

In the nurse rostering literature, problems are usually dealt with by constructing the schedules for each individual nurse, or by generating assignments of nurses to shifts for each day of the scheduling period. These approaches can be seen as decomposing the problems into sub-problems whose solutions are afterwards combined by considering the coverage constraints in Table 1 and constraints Nos. 24 and 25 in Table 2, or the other constraints in Tables 1 and 2, respectively.

Our approach decomposes problems in a different way. It consists of two stages. They are listed below and explained in more detail in Sects. 3.1 and 3.2: 
Table 3 Constraints for an example employee 1A

\begin{tabular}{|c|c|c|c|}
\hline & Constraints & Penalty & Details \\
\hline 1 & Max. number of assignments & 5 & Max. 19 shifts \\
\hline 2 & $\begin{array}{l}\text { Max. consecutive working } \\
\text { days }\end{array}$ & 10 & Max. 5 consecutive working days \\
\hline 3 & Min. consecutive working days & 10 & Min. 2 consecutive working days \\
\hline 4 & Min. consecutive free days & 10 & Min. 2 consecutive free days \\
\hline 5 & $\begin{array}{l}\text { Max. consecutive working } \\
\text { weekends }\end{array}$ & 40 & Max. 2 consecutive working weekends \\
\hline 6 & Complete weekends & 20 & $\begin{array}{l}\text { Weekends should either be free or work on all week- } \\
\text { end days }\end{array}$ \\
\hline 7 & $\begin{array}{l}\text { Identical shift types during } \\
\text { weekend }\end{array}$ & 5 & \\
\hline 8 & $\begin{array}{l}\text { Number of consecutive shift } \\
\text { types }\end{array}$ & 10 & $\begin{array}{l}\text { Valid lengths of sequences of consecutive shift types: } \\
\text { L: } 3,4 ; \mathrm{N}: 3,4 ; \mathrm{D}: 2,3,4,5 ; \mathrm{E}: 3,4 \text { (see definition in } \\
\text { Table 4) }\end{array}$ \\
\hline 9 & Max. shift types & 10 & $\begin{array}{l}\text { Max. } 8 \text { L shifts; Max. } 5 \text { N shifts; } \\
\text { Max. } 19 \text { D shifts; Max. } 8 \text { E shifts }\end{array}$ \\
\hline 10 & Max. hours worked & 1 & Max. 152 hours \\
\hline 11 & Shift type successions & 5 & $\begin{array}{l}\text { If shift type A is allowed to follow B the next day. } \\
\text { Allowed shift combinations: "E, E", "E, D", "E, L", } \\
\text { "E, N", "D, D", "D, L", "D, N", "L, L", "N, N" }\end{array}$ \\
\hline 12 & $\begin{array}{l}\text { No night shift before a free } \\
\text { weekend }\end{array}$ & 10 & A shift should not overlap with a free weekend \\
\hline
\end{tabular}

Table 4 Shift types referred to in employee 1A's constraints

\begin{tabular}{lll}
\hline Shift label & Shift type & Time period of the shift \\
\hline D & Day shift & $08: 00-16: 30$ \\
E & Early shift & $06: 30-14: 30$ \\
L & Late shift & $14: 00-22: 00$ \\
N & Night shift & $21: 30-07: 00$ \\
\hline
\end{tabular}

- A shift sequence's construction with respect to the sequence constraints in Tables 1 and 2; and

- A construction of schedules for the nurses which are combined into a roster with respect to the schedule and roster constraints in Tables 1 and 2.

The overall idea of our approach is to reduce the problem complexity by grouping the constraints into the two categories, each of which is considered separately in each stage of the solution approach. By constructing all the valid high quality shift sequences, and by constructing the roster based on these sequences only, the problems can be seen as being decomposed into two parts. Each part of the approach deals with a sub-problem with a much smaller search space than that of the original problem. 
Table 5 Example of some shift sequences for the example problem in Table 4

\begin{tabular}{lll}
\hline Shift sequence & Penalty & Comment \\
\hline D, D & 0 & \\
E, E, E & 0 & \\
D, D, E, E, E & 5 & E not allowed to follow D \\
L, L, L, D, D & 5 & D not allowed to follow L \\
N, N & 10 & Two N's not allowed \\
E, D, D & 10 & One E not allowed \\
D, D, E & 15 & E not allowed to follow D. One E not allowed \\
L, D, D & 15 & D not allowed to follow L. One L not allowed \\
L, N & 25 & N not allowed to follow L. One N not allowed. One L not allowed \\
D, E, E & 25 & E not allowed to follow D. One D not allowed. Two E's not allowed \\
D, E, D, E & 50 & E not allowed to follow D. One E not allowed. One D not allowed \\
\hline
\end{tabular}

\subsection{The construction of shift sequences}

In this stage of the approach, the best shift sequences are constructed for each nurse of different skills in each problem. They are collected and ranked by their penalties for easy retrieval in stage 2 of the approach (see Sect. 3.2). By systematically generating a large collection of all the possible permutations of shift types over relevant periods, all the possible shift sequences are enumerated for each nurse in each problem. The ones satisfying sequence constraint No. 1 in Table 1 (skill requirement) and with the lowest total penalty are collected. This set of shift sequences will not be changed in the later stage of the approach. Table 5 presents examples of some shift sequences generated for employee 1A, whose soft constraints are given in Table 3.

To decrease the complexity, we limit the number of possible valid shift sequences by either considering only sequences with a penalty below a certain threshold $(\leq 5$ or 10), or by selecting the best 50 sequences for each nurse in the second stage of the approach. The numbers are set by initial experiments and found to be applicable for all the benchmark problems presented in Sect. 4. For example, in Table 5, the sequence ' $E$ E E' is generated for employee $1 \mathrm{~A}$ with no penalty. The sequence 'D D E' in Table 5 has a penalty of 15 as $\mathrm{E}$ is not allowed to follow D (penalty 5) and a sequence of consecutive days of $\mathrm{E}$ shifts must be longer than two (penalty 10). For this employee, there are actually thirteen sequences (of maximum length five) with penalty zero; two with penalty five; thirty nine with penalty ten, twenty eight with penalty fifteen and so on.

Also, only sequences of length up to 5 are considered at this stage. Sequences could be longer, depending on the problems in hand. To construct sequences of length greater than 5, we combine these sequences of length up to 5 to produce new sequences. This is performed in the schedule and roster construction stage.

\subsection{The construction of schedules and a roster-based on shift sequences}

In the second stage of the approach, schedules for each nurse are constructed iteratively, using the shift sequences produced in stage 1, as described in Sect. 3.1, by 


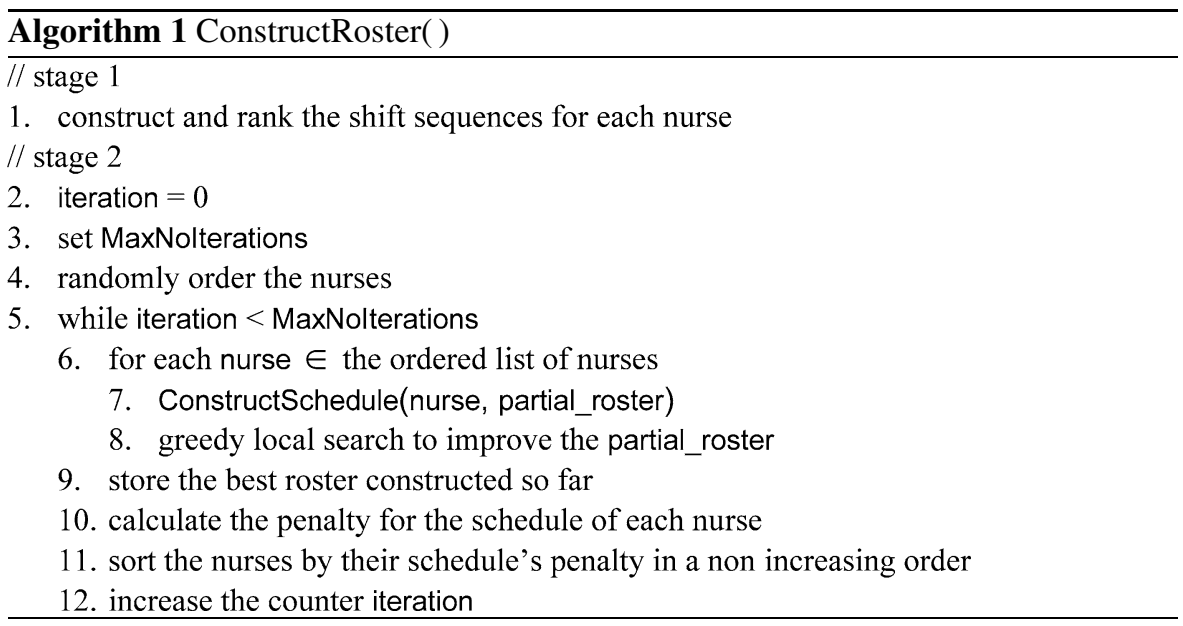

Algorithm 2 ConstructSchedule(nurse, partial_roster)

1. set final_threshold

2. current_threshold $=0$

3. while (current_threshold $\leq$ final_threshold)

4. for each shift sequence $\in$ the ranked list for the nurse do

5. for each day from the first day in the planning period

6. assign the sequence's corresponding shifts from this day based on the partial_roster, if it does not violate any hard constraint and the penalty occurred $<=$ current_threshold

7. increase the value of current_threshold by 5

8. return the schedule

considering the Schedule constraints for the particular nurse. Based on the schedules of nurses, the overall roster will be constructed by considering the Roster constraints in Sect. 3.1.

Algorithm 1 presents the pseudo-code for the overall two-stage approach for constructing the nurse schedules and overall roster. It is an adaptive iterative method where nurses that were difficult to schedule in previous iterations are scheduled first at the current iteration. That is, the nurses who received the highest schedule penalties in the last iteration are scheduled first.

Algorithm 2 presents the pseudo-code for the schedule construction process. It builds a schedule for the nurse based on the partial roster built so far for other nurses and returns its penalty to Algorithm 1. The overall guidance of Algorithm 2 is to generate a schedule with a low penalty value for the nurse, using low penalty shift sequences. In Algorithms 1 and 2, the schedule, sequence and roster constraints are combined (i.e. both are used) in penalty calculations and hard constraint violation checks. As Algorithm 1 is carried out iteratively while concerning the coverage requirement, and different orders of nurses are considered during iterations, the cost of the schedules for each nurse may be different at each iteration. Different shift 
sequences may be selected to construct different schedules, resulting in different rosters.

The basic idea is to assign as many shifts as possible to the most constrained nurse schedule, so as to obtain the lowest possible penalty. First, the shift sequences with the lowest penalty in the ranked list are considered. If no valid assignment can be made for the current nurse, the shift sequence with the second lowest penalty is considered and so on. The sequences are assigned for the current nurse if the penalty of assigning them is under the current threshold.

During the roster construction, and after a schedule has been generated for the current nurse, an improvement method based on an efficient greedy local search is carried out on the partial roster. It simply swaps any pair of shifts between two nurses in the (partial) roster, as long as the swaps satisfy hard constraints and decrease the roster penalty. By doing this, some of the days in the roster may be assigned shifts that cannot be made in the schedule construction. The method stops when no further improvement can be made. Then, the schedule for the next nurse selected is constructed based on the partial roster built so far, and improved by the greedy local search afterwards. The aim is to reduce the overall penalty of the partial rosters generated for all the nurses scheduled so far.

After all the schedules have been constructed and a roster has been built, there may still be some shifts for which the coverage is not satisfied. To repair this, a greedy heuristic is used. Each extra shift to be assigned is added to the nurse's schedule whose penalty decreases the most (or increases the least if all worsen) on receiving this shift. After this repair step, the local search is applied once more to improve the quality of the overall roster.

In real-world nurse rostering, the number of nurses needed in hospital wards is planned before the definition of the problem, i.e. the problem requires enough nurses of a certain skill level to satisfy the coverage requirement. For example, if there is a requirement for one head nurse shift on each day during the scheduling period, then at least one head nurse is included in the problem.

\section{Benchmark nurse rostering problems}

It is well known that in nurse rostering, researchers tend to deal with problems defined by local hospitals in different countries and, often, with simplifications of those problems (Burke et al. 2004c). This makes comparisons and evaluations of different approaches very difficult, if not impossible. In Burke et al. (2004c), it is stated that "it is extremely important to start building up easily accessible benchmark problems" in nurse rostering.

In this paper, we introduce a benchmark nurse rostering problem data set that consists of 11 different problem instances. The data sets were collected from rather small departments at real hospitals, using the nurse rostering model and algorithms developed at KaHo Sint-Lieven (Burke et al. 2004b). Before having made this data publicly available, we had to anonymize it, remove any confidential information and remove some rather country/location specific constraints in order to make the data more usable for other researchers. Obviously, this means that the data sets are slightly 
Table 6 Characteristics of the benchmark nurse rostering problem (Instances BCV-1.8.1, BCV-1.8.2 and BCV-1.8.3 are similar instances which differ in adjustments to some of the constraint parameters and to the personal requests)

\begin{tabular}{lccccccccccc}
\hline Problems & BCV- & BCV- & BCV- & BCV- & BCV- & BCV- & BCV- & BCV- & BCV- & BCV- & BCV- \\
& 1.8 .1 & 1.8 .2 & 1.8 .3 & 2.46 .1 & 3.46 .1 & 4.13 .1 & 5.4 .1 & 6.13 .1 & 7.10 .1 & 8.13 .1 & A.12.1 \\
\hline No. of nurses & 8 & 8 & 8 & 46 & 46 & 13 & 4 & 13 & 10 & 13 & 12 \\
No. of shift types & 4 & 4 & 4 & 3 & 3 & 4 & 4 & 4 & 6 & 4 & 4 \\
Period (days) & 28 & 28 & 28 & 28 & 26 & 29 & 28 & 30 & 28 & 28 & 31 \\
No. of skill levels & 2 & 2 & 2 & 1 & 1 & 2 & 1 & 2 & 1 & 2 & 2 \\
\hline
\end{tabular}

simpler than those presented in Burke et al. (2004b). Many of the instances are still far from trivial, however. The complexity loss is small as these problems retain most of the original constraints.

Table 6 presents the characteristics of the 11 problem instances in the data set. They are non-cyclic problems and are significantly different from each other, not only with respect to the number of nurses (ranging from 4 to 46), the number of skill levels ( 1 or 2 ) and the number of shift types, but also the number of personal requests (constraint 22 and 23 in Table 2) and the length of the scheduling period (ranging from 26 to 31 days). There are also different numbers of shift types (ranging from 3 to 6) in different problems. Note that the shift types in different problems may define different start and end times within a day. Rather than presenting specific constraints of fixed characteristics for particular problems, this set of benchmark data represents a wide variety of nurse rostering problems with non-trivial properties which are derived from complete real world complex instances.

The data set is available at http://www.asap.cs.nott.ac.uk/projects/nmhpr/data/ index.html, where problems are presented using XML, which is a very flexible, yet simple, format widely used in exchanging many types of data. The representation is easily extendable to add new characteristics and is flexible in presenting different problems in a variety of situations. At the same website, we also provide API functions that can be called to evaluate the quality of rosters as a standard measure to ensure the accuracy of any new result. The web site also presents additional details about the data sets.

\section{Experimental results}

We have tested the two-stage approach to solve the modified benchmark problem instances presented in Sect. 4. Two sets of experiments, with and without the adaptive ordering of nurses (step 11 in Algorithm 1), have been carried out. The results are presented in Tables 7 and 8, respectively. In each table, the results for two variants of the approach are reported: the approach reported in Algorithms 1 and 2; and the approach without the greedy local search (step 8 in Algorithm 1). When applying the algorithm, we resolved ties (when ordering the nurses with respect to schedule penalties and when selecting shift sequences in order of their quality) by creating a random order. For each problem instance, 8 runs with different seeds for the random 
Table 7 Results from the approach without adaptive ordering on the benchmark nurse rostering problems (GLS: Greedy Local Search; best results and best average are in bold)

\begin{tabular}{lrrrrrrrrrrr}
\hline Problems & BCV- & BCV- & BCV- & BCV- & BCV- & BCV- & BCV- & BCV- & BCV- & BCV- & BCV- \\
& 1.8 .1 & 1.8 .2 & 1.8 .3 & 2.46 .1 & 3.46 .1 & 4.13 .1 & 5.4 .1 & 6.13 .1 & 7.10 .1 & 8.13 .1 & A.12.1 \\
\hline With GLS best & 352 & 947 & $\mathbf{2 8 3}$ & $\mathbf{1 5 9 4}$ & $\mathbf{3 7 2 4}$ & $\mathbf{1 8}$ & $\mathbf{2 0 0}$ & $\mathbf{9 8 6}$ & 472 & $\mathbf{1 4 8}$ & $\mathbf{3 3 3 5}$ \\
With GLS worst & 378 & 1012 & 517 & 1680 & 3897 & 130 & 200 & 1528 & 596 & 160 & 4540 \\
With GLS avg & $\mathbf{3 6 5}$ & $\mathbf{9 7 5}$ & 347 & 1629 & $\mathbf{3 7 8 9}$ & 84 & $\mathbf{2 0 0}$ & $\mathbf{1 2 0 9}$ & 507 & 151 & $\mathbf{3 9 7 2}$ \\
Time (s) & 6 & 6 & 7 & 191 & 137 & 10 & 1 & 15 & 10 & 11 & 44 \\
Without GLS best & $\mathbf{3 2 3}$ & $\mathbf{9 3 0}$ & 315 & 1603 & 3795 & 19 & $\mathbf{2 0 0}$ & 1251 & $\mathbf{4 2 7}$ & $\mathbf{1 4 8}$ & 3845 \\
Without GLS worst & 537 & 1134 & 347 & 1646 & 3911 & 155 & 200 & 1519 & 543 & 153 & 4688 \\
Without GLS avg & 414 & 1024 & $\mathbf{3 3 1}$ & $\mathbf{1 6 2 6}$ & 3861 & $\mathbf{8 1}$ & $\mathbf{2 0 0}$ & 1361 & $\mathbf{4 8 9}$ & $\mathbf{1 4 9}$ & 4191 \\
Time (s) & 3 & 2 & 2 & 21 & 21 & 3 & 0 & 4 & 4 & 3 & 13 \\
\hline
\end{tabular}

Table 8 Results from the approach with adaptive ordering on the benchmark nurse rostering problems (GLS: Greedy Local Search; best results and best average are in bold)

\begin{tabular}{lrrrrrrrrrrr}
\hline Problems & BCV- & BCV- & BCV- & BCV- & BCV- & BCV- & BCV- & BCV- & BCV- & BCV- & BCV- \\
& 1.8 .1 & 1.8 .2 & 1.8 .3 & 2.46 .1 & 3.46 .1 & 4.13 .1 & 5.4 .1 & 6.13 .1 & 7.10 .1 & 8.13 .1 & A.12.1 \\
\hline With GLS best & $\mathbf{3 6 2}$ & 942 & $\mathbf{2 8 8}$ & $\mathbf{1 5 9 6}$ & $\mathbf{3 6 0 1}$ & $\mathbf{2 1}$ & $\mathbf{2 0 0}$ & 1037 & $\mathbf{3 9 6}$ & $\mathbf{1 4 8}$ & $\mathbf{3 5 7 0}$ \\
With GLS worst & 413 & 1023 & 383 & 1620 & 3761 & 130 & 200 & 1544 & 601 & 153 & 4450 \\
With GLS avg & $\mathbf{3 8 8}$ & 974 & $\mathbf{3 3 0}$ & $\mathbf{1 6 0 9}$ & $\mathbf{3 7 0 5}$ & $\mathbf{6 6}$ & $\mathbf{2 0 0}$ & $\mathbf{1 3 3 4}$ & 540 & $\mathbf{1 5 0}$ & $\mathbf{3 8 5 6}$ \\
Time (s) & 6 & 6 & 6 & 196 & 184 & 10 & 1 & 15 & 10 & 11 & 47 \\
Without GLS best & 363 & $\mathbf{9 2 9}$ & 314 & 1606 & 3669 & 67 & $\mathbf{2 0 0}$ & $\mathbf{8 9 0}$ & $\mathbf{3 9 6}$ & $\mathbf{1 4 8}$ & 3743 \\
Without GLS worst & 546 & 997 & 483 & 1636 & 3894 & 107 & 200 & 1584 & 586 & 160 & 4908 \\
Without GLS avg & 431 & $\mathbf{9 6 4}$ & 353 & 1624 & 3804 & 76 & $\mathbf{2 0 0}$ & 1346 & $\mathbf{4 6 7}$ & 152 & 4239 \\
Time (s) & 2 & 2 & 2 & 20 & 19 & 3 & 0 & 4 & 3 & 3 & 14 \\
\hline
\end{tabular}

generator are carried out, of which the best, worst and average value of the results are reported. The best results are highlighted in bold. The experiments are carried out on a Pentium IV $2.4 \mathrm{GHz}$ machine.

We can see from both Tables 7 and 8 that greedy local search during the roster construction greatly improves the solution quality. The adaptive ordering of nurses during the iterative process also has a positive effect on the results of the construction approach (the results in Table 8 are generally better than those in Table 7). This can be seen in Table 9, where the average improvements using greedy local search and adaptive ordering are presented.

Note that, without the greedy local search heuristic, the approach is purely constructive. It obtains the result fairly quickly (within 21 seconds for the largest instances). Moreover, it does not require much fine-tuning of the parameters when dealing with specific problems. The only parameter that can be changed is the increment value of current_threshold. All the problem instances (having very different characteristics) have been solved using the same constructive method. 
Table 9 Average \% improvements from greedy local search or adaptive ordering over all data sets

\begin{tabular}{lll}
\hline & $\begin{array}{l}\text { Average \% improvement using } \\
\text { GLS compared with no GLS }\end{array}$ & $\begin{array}{l}\text { Average \% improvement using adaptive ordering } \\
\text { compared with no adaptive ordering }\end{array}$ \\
\hline Best case & 3.8 & 1.7 \\
Worst case & 3.4 & 0.3 \\
Average & 3.7 & 0.5 \\
\hline
\end{tabular}

\section{Conclusions and future work}

In this paper, we introduced a set of benchmark nurse rostering problems and reported the first results on this data. To encourage scientific comparisons we have set up a web site at http://www.asap.cs.nott.ac.uk/projects/nmhpr/data/index.html, where $\mathrm{XML}$ is used to present different problems. The representation can be easily extended to include new constraints. So either similar or very different instances can be easily introduced. We also provide a standard API evaluation function and updated solutions for the problems whenever newly published results become available. The aim is to enable easy scientific comparisons on the complex nurse rostering problems with a variety of constraints. We welcome more nurse rostering research upon this data set.

We investigated a constructive approach where constraints in nurse rostering problems are grouped into sequence, schedule and roster constraints. Each of the groups is dealt with respectively in constructing shift sequences, constructing schedules for nurses based on the sequences obtained, and constructing the overall roster. The problem complexity can thus be considerably reduced as problems can be seen as decomposed into two parts, each of which is only concerned with the related constraints and thus is much smaller.

When constructing the schedules, the algorithm adaptively orders and selects nurses according to the difficulty of constructing their schedules in the previous iteration. Nurses that received high penalty schedules in previous iterations of the schedule construction will be considered early in the current iteration, which reduces the chance of having trouble with generating their individual schedule later on in the process. The approach is simple and efficient. It obtains reasonably good results quickly for a set of real world, newly presented benchmark nurse rostering problems. It is purely constructive and does not involve much fine-tuning effort on parameters for different problems. The adaptive selection is a robust strategy that does not rely on any domain knowledge.

It is also observed that the greedy local search carried out between the schedule constructions for each nurse greatly improved the roster constructed. This indicates that the approach can be easily adapted for hybridisation with other techniques (i.e. exact methods and meta-heuristics). It will be particularly interesting to perform a study using exact methods to obtain the best combinations for high quality schedules or rosters. Operating on sequences, rather than on individual shifts by meta-heuristics, is also an interesting direction for future work. More strategies of adaptive selection of nurses and sequences will be explored, employing new general methodologies such as hyper-heuristics (e.g. Burke et al. 2003a, 2003b). 
Acknowledgements This work was supported by EPSRC grants GR/S31150/01 and GR/T23374/01. We would like to thank for the anonymous referees for their constructive comments and suggestions.

\section{References}

Adbennadher, S., Schlenker, H.: Nurse scheduling using constraint logic programming. In: Eleventh Annual Conference on Innovative Applications of Artificial Intelligence, IAAI-99, pp. 838-843, July 1999, Orlando, Florida, USA

Aickelin, U., Dowsland, K.: Exploiting problem structure in a genetic algorithm approach to a nurse rostering problem. J. Sched. 3(3), 139-153 (2000)

Aickelin, U., Dowsland, K.: An indirect genetic algorithm for a nurse scheduling problem. J. Oper. Res. Soc. 31(5), 761-778 (2003)

Aickelin, U., Li, J.: An estimation of distribution algorithm for nurse scheduling. Ann. Oper. Res. 155(1), 289-309 (2007)

Bard, J., Purnomo, H.W.: Preference scheduling for nurses using column generation. Eur. J. Oper. Res. 164, 510-534 (2005)

Bard, J., Purnomo, H.W.: Cyclic preference scheduling of nurses using a Lagrangian-based heuristic. J. Sched. 10(1), 5-23 (2007)

Beaumont, N.: Scheduling staff using mixed integer programming. Eur. J. Oper. Res. 98, 473-484 (1997)

Beddoe, G., Petrovic, S.: Determining feature weights using a genetic algorithm in a case-based reasoning approach to personnel rostering. Eur. J. Oper. Res. 175, 649-671 (2006)

Brusco, M.J., Jacobs, L.W.: Cost analysis of alternative formulations for personnel scheduling in continuously operating organisations. Eur. J. Oper. Res. 86, 249-261 (1995)

Brucker, P., Qu, R., Burke, E.K., Post, G.: A decomposition, construction and post-processing approach for a specific nurse rostering problem. In: Kendall, G., Lei, L., Pinedo, M. (eds.) Proceeding of the 2nd Multidisciplinary International Conference on Scheduling: Theory and Applications (MISTA'05), pp. 397-406, New York, USA, July 2005

Burke, E.K., De Causmaecker, P., Vanden Berghe, G.: A hybrid tabu search algorithm for the nurse rostering problem. In: Selected Papers from the 2nd Asia Pacific Conference on Simulated Evolution and Learning (SEAL'98). Lecture Notes in Artificial Intelligence, vol. 1585, pp. 187-194. Springer, Berlin (1999)

Burke, E.K., Cowling, P., De Causmaecker, P., Vanden Berghe, G.: A memetic approach to the nurse rostering problem. Appl. Intell. 15, 119-214 (2001)

Burke, E.K., Hart, E., Kendall, G., Newall, J., Ross, P., Schulenburg, S.: Hyperheuristics: an emerging direction in modern search technology. In: Glover, F., Kochenberger, G. (eds.) Handbook of Metaheuristics, pp. 457-474. Kluwer, Dordrecht (2003a)

Burke, E.K., Kendall, G., Soubeiga, E.: A tabu-search hyperheuristic for timetabling and rostering. J. Heur. 9(6), 451-470 (2003b)

Burke, E.K., De Causmaecker, P., Petrovic, S., Vanden Berghe, G.: Variable neighborhood search for nurse rostering problems. In: Resende, M.G.C., Pinho de Sousa, J.P. (eds.) Metaheuristics: Computer Decision-Making. Combinatorial Optimization Book Series, pp. 153-172. Kluwer, Dordrecht (2004a). Chapter 7

Burke, E.K., De Causmaecker, P., Vanden Berghe, G.: Novel meta-heuristic approaches to nurse rostering problems in Belgian hospitals. In: Leung, J. (ed.) Handbook of Scheduling: Algorithms, Models and Performance Analysis, pp. 44.1-44.18. CRC Press, Boca Raton (2004b). Chapter 44

Burke, E.K., De Causmaecker, P., Vanden Berghe, G., Van Landeghem, H.: The state of the art of nurse rostering. J. Sched. 7(6), 441-499 (2004c)

Burke, E.K., Curtois, T., Post, G., Qu, R., Veltman, B.: A hybrid heuristic ordering and variable neighbourhood search for the nurse rostering problem. Eur. J. Oper. Res. 2, 330-341 (2008)

Chen, J.G., Yeung, T.: Hybrid expert system approach to nurse scheduling. Comput. Nursing 11(4), 183192 (1993)

Dowsland, K.: Nurse scheduling with tabu search and strategic oscillation. Eur. J. Oper. Res. 106, 393-407 (1998)

Ernst, A.T., Jiang, H., Krishnamoorthy, M., Sier, D.: Staff scheduling and rostering: a review of applications, methods and models. Eur. J. Oper. Res. 153, 3-27 (2004)

Ikegami, A., Niwa, A.: A subproblem-centric model and approach to the nurse scheduling problem. Math. Program. 97(3), 517-541 (2003) 
Jan, A., Yamamoto, M., Ohuchi, A.: Evolutionary algorithms for nurse scheduling problem. In: Proceedings of the 2000 Congress on Evolutionary Computation (CEC'00), pp. 196-203, San Diego, USA, 2000

Jaszkiewicz, A.: A metaheuristic approach to multiple objective nurse scheduling. Found. Comput. Decis. Sci. 22(3), 169-184 (1997)

Jaumard, B., Semet, F., Vovor, T.: A generalized linear programming model for nurse scheduling. Eur. J. Oper. Res. 107, 1-8 (1998)

Mason, A., Smith, M.: A nested column generator for solving rostering problems with integer programming. In: Caccetta, L., Teo, K.L., Siew, P.F., Leung, Y.H., Jennings, L.S., Rehbock, V. (eds.) Proceedings of the 4th International Conference on Optimisation: Techniques and Applications, pp. 827-834. July 1998, Perth, Australia

Meisels, A., Gudes, E., Solotorevsky, G.: Employee timetabling, constraint networks and knowledge-based rules: a mixed approach. In: Burke, E.K., Ross, P. (eds.) Selected Papers from the 1st International Conference on Practice and Theory of Automated Timetabling. Lecture Notes in Computer Science, vol. 1153, pp. 93-105. Springer, Berlin (1996)

Meyer auf'm Hofe, H.: Solving rostering tasks as constraint optimisation. In: Burke, E.K., Erben, W. (eds.) Selected Papers from the 3rd International Conference on Practice and Theory of Automated Timetabling. Lecture Notes in Computer Science, vol. 2079, pp. 280-297. Springer, Berlin (2000)

Millar, H.H., Kiragu, M.: Cyclic and non-cyclic scheduling of $12 \mathrm{~h}$ shift nurses by network programming. Eur. J. Oper. Res. 104, 582-592 (1998)

Petrovic, S., Beddoe, G., Vanden Berghe, G.: Storing and adapting repair experiences in personnel rostering. In: Burke, E.K., De Causmaecker, P. (eds.) Selected Papers from the 4th International Conference on Practice and Theory of Automated Timetabling. Lecture Notes in Computer Science, vol. 2740, pp. 148-165. Springer, Berlin (2003)

Pierskalla, W., Rath, G.: Nurse scheduling using mathematical programming. Oper. Res. 24(5), 857-870 (1976)

Post, G., Veltman, B., Harmonious personnel scheduling. In: Burke, E.K., Trick, M. (eds.) Proceedings of the 5th International Conference on the Practice and Automated Timetabling, pp. 557-559. August 2004, Pittsburgh, PA, USA

Scott, S., Simpson, R.M.: Case-based incorporating scheduling constraint dimensions: experiences in nurse rostering. In: Smyth, Cunningham (eds.) Advances in Case Based Reasoning. Lecture Notes in Artificial Intelligence, vol. 1488, pp. 392-401. Springer, Berlin (1998)

Sitompul, D., Randhawa, S.: Nurse rostering models: a state-of-the-art review. J. Soc. Health Syst. 2(1), 62-72 (1990)

Valouxis, C., Housos, E.: Hybrid optimisation techniques for the workshift and rest assignment of nursing personnel. Artif. Intell. Med. 20, 155-175 (2000)

Warner, M., Keller, B., Martel, S.: Automated nurse scheduling. J. Soc. Health Syst. 2(2), 66-80 (1990) 